\title{
Congresses, conferences, workshops and courses
}

(C) Springer-Verlag GmbH Germany, part of Springer Nature 2018

January 2019

21 January 2019

Tel Aviv, Israel

International Workshop "Quality of Test Results for Conformity

Assessment of a Chemical Composition - What is Good and What is Bad?"

http://www.isranalytica.org.il

e-mail: reutl@bioforum.co.il

22-23 January 2019

Tel Aviv, Israel Isranalytica Conference and Exhibition http://www.isranalytica.org.il e-mail: reut@bioforum.co.il
June 2019

18-20 June 2019

The Hague, The Netherlands

GAS Analysis 2019

http://www.gasanalysisevent.com/ e-mail: gas@nen.nl 